Biochimica et Biophysica Acta, 497 (1977) 260-271

(C) Elsevier/North-Holland Biomedical Press

BBA 28180

\title{
MOLECULAR MOBILITIES OF SOLUBLE COMPONENTS IN THE AQUEOUS PHASE OF CHROMAFFIN GRANULES
}

\author{
ROBERT R. SHARP and EDWARD P. RICHARDS \\ Department of Chemistry, The University of Michigan, Ann Arbor, Mich. 48104 (U.S.A.)
}

(Received August 17th, 1976)

\section{Summary}

NMR relaxation times have been used to characterize molecular motion and intermolecular complexes in the aqueous phase of bovine chromaffin granules. Partially relaxed ${ }^{13} \mathrm{C}$ and proton spectra have been obtained at 3 and $25^{\circ} \mathrm{C} . T_{1}$ measurements of five protonated carbons on epinephrine $\left(\mathrm{C}_{2}, \mathrm{C}_{5}, \mathrm{C}_{6}, \mathrm{CHOH}\right.$ and $\left.\mathrm{NCH}_{3}\right)$ give a correlation time of $0.15\left(10^{-9}\right) \mathrm{s}$ at $25^{\circ} \mathrm{C}$ for the catechol ring and methine carbon, while the effective correlation time for the $\mathrm{NCH}_{3}$ group is somewhat shorter due to its internal degree of rotational freedom. Resonances of protonated carbons on the soluble protein chromogranin give very similar correlation times: $0.20\left(10^{-9}\right) \mathrm{s}$ for the peptide $\alpha$-carbon and 0.2 $\left(10^{-9}\right) \mathrm{s}$ for the methylene sidechain carbons of glutamic acid. The correlation time $\left(\tau_{\mathrm{R}}\right)$ of ATP was not measured directly using ${ }^{13} \mathrm{C} T_{1}$ data due to the weakness of its spectrum, but its reorientation appears to be substantially slower than that of epinephrine or chromogranin. This conclusion is based on three observations: (1) the qualitative temperature dependence of $T_{1}$ for $\mathrm{H}_{2}$ and $\mathrm{H}_{8}$ on the adenine ring places $\tau_{\mathrm{R}}$ for ATP to the right of the $T_{1}$ minimum, or $\tau_{\mathrm{R}} \geqslant$ $1.0\left(10^{-9}\right) \mathrm{s} ;(2){ }^{13} \mathrm{C}$ resonances of ATP have anomalously low amplitudes compared with epinephrine resonances, a fact that is readily explained only if ATP undergoes substantially slower reorientation; and (3) a comparison of the $T_{1}$ data of $\mathrm{H}_{8}$ in chromaffin granules and in a dilute aqueous solution, where $\tau_{\mathrm{R}}$ for ATP can be measured directly, indicates that $\tau_{\mathrm{R}} \approx 1.0\left(10^{-9}\right) \mathrm{s}$ at $25^{\circ} \mathrm{C}$ in the granules. The relaxation data are consistent with the concept of a storage complex based on electrostatic interactions between a polyion (chromogranin) and its counterions (ATP and epinephrine), in which ATP cross-links cationic sidechains of the protein.

\section{Introduction}

High resolution NMR spectra of the ${ }^{1} \mathrm{H},{ }^{13} \mathrm{C}$ and ${ }^{31} \mathrm{P}$ resonances of intact chromaffin granules have recently been described $[1,2]$. These spectra demon- 
strate the presence of a fairly fluid, essentially isotropic aqueous phase in the vesicle interior. This phase contains primarily epinephrine, chromogranin and ATP with little, if any, lipid. The nature of the intermolecular interactions among the soluble components has not been elucidated, although some form of complex appears necessary to explain the well-known stability of the granules with respect to epinephrine efflux [3-8]. Although they provide little direct information about the nature of the complex, the NMR spectra do provide several intriguing clues.

First, the $\gamma^{-31} \mathrm{P}$ of ATP, and to a lesser extent the $\beta{ }^{31} \mathrm{P}$, are shifted downfield in the vesicles as would be consistent with ionic complexation at the phosphate esters. Second, a comparison of relative intensities in the ${ }^{13} \mathrm{C}$ spectra suggests that epinephrine and ATP have significantly different rotational correlation times in the granules and consequently are not present as a simple binary complex. Thirdly, chromogranin is present as a highly distended random coil polyelectrolyte. The protein does not form a gel, but probably interacts strongly with other ions in the aqueous phase (primarily epinephrine and ATP) through polyelectrolyte-counterion interactions.

In order to probe more directly the molecular nature of complexes in the aqueous phase we have measured ${ }^{13} \mathrm{C}$ and ${ }^{1} \mathrm{H}$ spin-lattice relaxation times $\left(T_{1}\right.$ data) in the granules. $T_{1}$ data provide a direct measure of the correlation times for molecular reorientation experienced by a variety of nuclei on different solute molecules. Thus, these measurements provide a detailed picture of molecular motion in the vesicle interior. A comparison of correlation times of nuclei on different molecules also provides a useful criterion of intermolecular complexation.

The theory of spin-lattice relaxation is discussed in detail elsewhere [9]. $T_{1}$ is in general a double-valued function of the reorientational correlation time, $\tau_{R}$, and can be written $T_{1}^{-1}=\overline{\phi^{2}} f_{1}\left(\tau_{R}\right)$. In the systems of interest here, relaxation results from coupling between neighboring nuclear magnetic dipoles, and $\overline{\phi^{2}}$ is the mean squared dipolar coupling energy. $f_{1}\left(\tau_{\mathrm{R}}\right)$ is proportional to the Fourier amplitudes of the (time-dependent) dipolar interaction at the Zeeman transition frequencies. Proton $T_{1}$ data result from both intra- and intermolecular dipolar couplings. In the present experiments, the intermolecular contribution is suppressed by exchanging the solvent with ${ }^{2} \mathrm{H}_{2} \mathrm{O}$. The intramolecular contribution is given by

$$
\begin{gathered}
\left(T_{\mathrm{l}}\right)^{-1}=2 \gamma_{\mathrm{H}}^{4} \hbar^{2} I(I+1) \sum_{j} r_{i j}^{-6} f_{1}^{1}\left(\tau_{\mathrm{R}}\right) \\
=5.39\left(10^{59}\right) \sum_{j} r_{i j}^{-6} f_{1}\left(\tau_{\mathrm{R}}\right),
\end{gathered}
$$

where

$f_{1}^{\mathrm{H}}\left(\tau_{\mathrm{R}}\right)=\frac{1}{5}\left\{\frac{\tau_{\mathrm{R}}}{1+\omega_{\mathrm{H}}^{2} \tau_{\mathrm{R}}^{2}}+\frac{4 \tau_{\mathrm{R}}}{1+4 \omega_{\mathrm{H}}^{2} \tau_{\mathrm{R}}^{2}}\right\}$

$\gamma_{\mathrm{H}}$ is the proton gyromagnetic ratio, $I$ is the nuclear spin quantum number, $r_{i j}$ is an internuclear distance, and $\omega_{H}$ is the proton Larmor frequency. The functional dependence of $T_{1}$ on $\tau_{\mathrm{R}}$ is plotted in Fig. 5 . The transverse relaxation 
time $T_{2}$, is also plotted in the figure. $T_{2}$ is conveniently measured from the linewidth, $W=\left(\pi T_{2}\right)^{-1}$ and is discussed fully in ref. 2. $T_{1}$ passes through a minimum in the neighborhood of $4 \omega_{\mathrm{H}}^{2} \tau_{\mathrm{R}}^{2}=1$, and becomes proportional to $\tau_{\mathrm{R}}$ to the right of this minimum. Thus, $T_{1}$, unlike $T_{2}$, is not a monotonically decreasing function of $\tau_{\mathrm{R}}$; and unlike $T_{2}, T_{1}$ is not sensitive to anisotropy in the local molecular environment that is associated with phase boundaries in heterogeneous media. Eqns. 1 assume that the medium is reasonably fluid $\left(\tau_{\mathrm{R}} \leqslant 10^{-5} \mathrm{~s}\right)$, and that the motions of all nuclei $i, j$ that contribute to the coupling are described by a single correlation time. For functional groups with internal rotational degrees of freedom, such as unhindered methyl groups, Eqn. 1 gives an effective correlation time which is always shorter than $\tau_{\mathrm{R}}$. For methyl groups, $\tau_{\text {eff }}$ lies in the range $\tau_{\mathrm{R}} \geqslant \tau_{\text {eff }} \geqslant \tau_{\mathrm{R}} / 4$, where the lower limit corresponds to completely free internal rotation [10].

$T_{1}$ data of ${ }^{13} \mathrm{C}$ nuclei that are directly bonded to protons are strongly dominated by the nearest neighbor ${ }^{13} \mathrm{C}-{ }^{1} \mathrm{H}$ interaction. For a ${ }^{13} \mathrm{C}$ nucleus directly bonded to $\mathrm{N}$ hydrogens $[9,11]$

$$
\begin{gathered}
\left(T_{1}\right)^{-1}=\frac{N \gamma_{\mathrm{C}}^{2} \gamma_{\mathrm{H}}^{2} \hbar^{2}}{r_{\mathrm{CH}}^{6}} f_{1}^{\mathrm{C}}\left(\tau_{\mathrm{R}}\right) \\
=2.40\left(10^{10}\right) N f_{1}^{\mathrm{C}}\left(\tau_{\mathrm{R}}\right)
\end{gathered}
$$

where

$f_{1}^{\mathrm{C}}\left(\tau_{\mathrm{R}}\right)=\frac{1}{10}\left\{\frac{\tau_{\mathrm{R}}}{1+\left(\omega_{\mathrm{C}}-\omega_{\mathrm{H}}\right)^{2} \tau_{\mathrm{R}}^{2}}+\frac{3 \tau_{\mathrm{R}}}{1+\omega_{\mathrm{C}}^{2} \tau_{\mathrm{R}}^{2}}+\frac{6 \tau_{\mathrm{R}}}{1+\left(\omega_{\mathrm{C}}+\omega_{\mathrm{H}}\right)^{2} \tau_{\mathrm{R}}^{2}}\right\}$

The carbon-proton bond length is taken to be $1.09\left(10^{-8}\right) \mathrm{cm}$ in Eqn. 2. Plots of $T_{1}$ and $T_{2}$ vs. $\tau_{\mathbf{R}}$ for ${ }^{13} \mathrm{C}$ are shown in Fig. 3. Doddrell et al. [11] have computed the effects of internal rotation on ${ }^{13} \mathrm{C} T_{1}$ data. In the extreme narrowing limit, which is characterized by $\left(\omega_{\mathrm{C}}+\omega_{H}\right)^{2} \tau_{\mathrm{R}}^{2}<<1$, free internal rotation reduces $\tau_{\text {eff }}$ of a methyl carbon to $1 / 9$ of the value, $\tau_{R}$, that corresponds to no internal rotation.

\section{Experimental}

Chromaffin granules were prepared by differential centrifugation from homogenized bovine adrenal medullae as described previously [2]. All samples were prepared and kept at $3 \pm 2^{\circ} \mathrm{C}$ until used. Reference samples containing epinephrine (1.0 M in $\left.{ }^{2} \mathrm{H}_{2} \mathrm{O}, \mathrm{p}^{2} \mathrm{H} 7.2\right)$ and ATP $\left(0.5 \mathrm{M}\right.$ in $\left.{ }^{2} \mathrm{H}_{2} \mathrm{O}, \mathrm{p}^{2} \mathrm{H} 7.2\right)$ were prepared by weight and degassed by several cycles of the freeze-pump-thaw method.

NMR spectra were obtained from a JEOL JNM-PFT/100 Fourier transform NMR spectrometer interfaced to two different data systems. A Digilab NMR-3 data system was used initially to obtain a complete set of data $\left({ }^{1} \mathrm{H}\right.$ at $3^{\circ} \mathrm{C}, 25^{\circ} \mathrm{C}$ and ${ }^{13} \mathrm{C}$ at $3^{\circ} \mathrm{C}, 25^{\circ} \mathrm{C}$ ). Subsequently, the spectrometer was interfaced to a JEOL EC-100 computer system and a crystal filter was added, resulting in a substantial improvement in signal-to-noise factor. The proton measurements at 3 and $25^{\circ} \mathrm{C}$ and the ${ }^{13} \mathrm{C}$ measurements at $25^{\circ} \mathrm{C}$ were then repeated. 
Relaxation times were determined from partially relaxed $\left(180^{\circ}-\tau-90^{\circ}\right.$ sample $)_{n}$ spectra. Delays of $1.2-2.0 \mathrm{~s}$ were inserted between successive sequences in the ${ }^{13} \mathrm{C}$ measurements. These delays ensure essentially complete relaxation of all protonated carbons but not necessarily of the non-protonated carbons. Delays in the proton measurements were at least five times the longest $T_{1}$ in the spectrum. $90^{\circ}$ pulse widths for both ${ }^{1} \mathrm{H}$ and ${ }^{13} \mathrm{C}$ were approx. $22 \mu \mathrm{s}$.

${ }^{13} \mathrm{C} T_{1}$ data at $25^{\circ} \mathrm{C}$ have been computed from semilogarithmic plots of amplitude against time for five of the epinephrine-protonated carbons $\left(\mathrm{C}_{2}, \mathrm{C}_{5}, \mathrm{C}_{6}\right.$, $\mathrm{CHOH}, \mathrm{NCH}_{3}$ ); typical least-square plots of the data are shown in Fig. 2. Data at $3^{\circ} \mathrm{C}$ were obtained without using a crystal filter and the spectra therefore have somewhat poorer signal-to-noise ratio. $T_{1}$ data at this temperature were computed from the null points, using $T_{1}=t_{\text {nul1 }} / \ln 2$. This procedure gives greater uncertainty in $T_{1}$ but is free from systematic error that can arise from an inaccurate value of the amplitude at $t_{\infty}$. Proton $T_{1}$ data were also computed from the null points, in this case because spectral overlap is relatively severe due to the much smaller magnitudes of proton chemical shifts. Null points of neighboring peaks can readily be estimated in the presence of moderate overlap that would cause systematic baseline error in semi-logarithmic plots. Estimated uncertainties in $T_{1}$ depend on peak intensity as well as on the degree of overlap, and are given in Table II.

\section{Results}

Partially relaxed ${ }^{13} \mathrm{C}$ spectra of isolated chromaffin granules at $25^{\circ} \mathrm{C}$ are shown in Fig. 1. A complete assignment of the carbon spectrum is given in ref. 2; the peaks of protonated carbons, which were used for the measurement of correlation times, are identified for convenience in the figure. The longest relaxation times are found for non-protonated carbons (denoted by dashed lines in the figure) in the downfield region. Among the epinephrine carbons, $C_{1}$ at $133 \mathrm{ppm}$ and $\left(\mathrm{C}_{3}, \mathrm{C}_{4}\right)$, which are accidentally degenerate at $145 \mathrm{ppm}$, have somewhat longer $T_{1}$ data than do any of the protonated carbons, which include $\mathrm{C}_{2}, \mathrm{C}_{5}, \mathrm{C}_{6}$, $\mathrm{CHOH}$, and $\mathrm{NCH}_{3}$. Similarly chromogranin resonances in the amide region near $178 \mathrm{ppm}$ relax considerably more slowly than does the intense peak due to $\alpha$-methine carbons of the protein backbone or any of the peaks in the sidechain methylene region. On the other hand, $T_{1}$ data of the protonated carbons of epinephrine are very similar to $T_{1}$ data of the protonated carbons of chromogranin, including the backbone methine carbons, indicating general similarity of the rotational correlation times of these molecules. Unfortunately, the ATP resonances are of anomalously low amplitude in the carbon spectra and are obscured by the baseline noise. As pointed out in ref. 2, this intensity loss would be expected if the correlation time for ATP were substantially longer than those for epinephrine or chromogranin.

${ }^{13} \mathrm{C} T_{1}$ data have been measured for five of the epinephrine protonated carbons $\left(\mathrm{C}_{2}, \mathrm{C}_{5}, \mathrm{C}_{6}, \mathrm{CHOH}\right.$ and $\left.\mathrm{NCH}_{3}\right)$ and are listed in Table I. Correlation times corresponding to these values have been obtained by plotting the $T_{1}$ data on theoretical curves of Eqn. 2 which are shown in Fig. 3.

Since $T_{1}$ is a double-valued function of $\tau_{\mathrm{R}}$, an experimental criterion is necessary to decide whether the correlation time lies to the left or right of the $T_{1}$ 


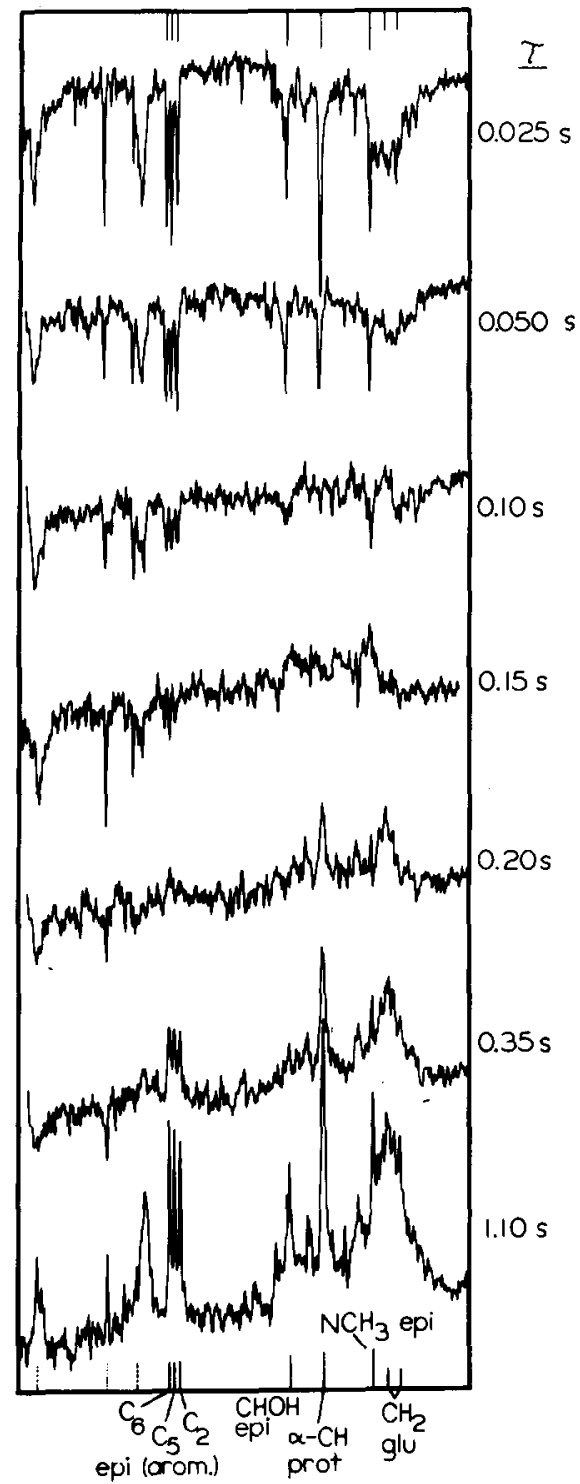

Fig. 1. Partially relaxed ${ }^{13} \mathrm{C}$ spectra of isolated chromaffin granules at $25^{\circ} \mathrm{C}$. Assignments are given for protonated carbons used in the analysis of correlation times. Dashed lines denote non-protonated carbons: (from left to right) the amide carbon region of chromogranin, $\left(C_{3}, C_{4}\right)$ of epinephrine and $C_{1}$ of epinephrine.

minimum. Appropriate criteria are provided by two independent observations. First the ${ }^{13} \mathrm{C}$ linewidths, which are reported in ref. 2, place an absolute upper limit on $\tau_{R}$. These limiting values, which are obtained by neglecting the effect of motional anisotropy on the linewidth, demonstrate that $\tau_{R} \leqslant 2$ ns for all epinephrine carbons and that $\tau_{R}<7$ ns for the chromogranin backbone [2]. Thus, the solution of Eqn. 2 for which $\tau_{\mathrm{R}} \approx 200 \mathrm{~ns}$ is clearly excluded. Incidentally, this comparison also shows that $T_{2}$ in the aqueous phase is strongly dominated by effects of motional anisotropy due to the influence of phase bound- 
TABLE I

${ }^{3} \mathrm{C} T_{1}$ DATA AND CORRELATION TIMES IN CHROMAFFIN GRANULES

\begin{tabular}{|c|c|c|c|c|c|}
\hline \multirow[t]{2}{*}{ Molecule } & \multirow[t]{2}{*}{ Carbon } & \multicolumn{2}{|l|}{$3^{\circ} \mathrm{C}$} & \multicolumn{2}{|l|}{$25^{\circ} \mathrm{C}$} \\
\hline & & $T_{1}(\mathrm{~s})$ & $\tau_{R}(\mathrm{~ns})$ & $T_{1}(\mathbf{s})$ & $\tau_{\mathrm{R}}(\mathrm{ns})$ \\
\hline \multirow[t]{5}{*}{ Epinephrine } & $\mathrm{C}-2$ & $0.14 \pm 0.06$ & 0.32 & $0.31 \pm 0.04$ & 0.15 \\
\hline & C-5 & $0.14 \pm 0.06$ & 0.32 & $0.31 \pm 0.04$ & 0.15 \\
\hline & C-6 & $0.14 \pm 0.06$ & 0.32 & $0.34 \pm 0.04$ & 0.135 \\
\hline & ${ }^{13} \mathrm{CHOH}$ & $0.14 \pm 0.05$ & 0.32 & $0.25 \pm 0.04$ & 0.18 \\
\hline & $\mathrm{N}^{13} \mathrm{CH}_{3}$ & $0.29 \pm 0.10$ & $0.053^{\mathrm{a}}$ & $0.32 \pm 0.06$ & $0.047^{\mathrm{a}}$ \\
\hline \multirow[t]{3}{*}{ Chromogranin } & $\alpha-\mathrm{CH}$ & $0.12 \pm 0.04$ & 0.37 & $0.23 \pm 0.03$ & 0.20 \\
\hline & Glu $-\beta \mathrm{CH}_{2}$ & & & $0.07-0.20$ & $0.12-0.33$ \\
\hline & Glu- $\gamma \mathrm{CH}_{2}$ & & & $0.07-0.20$ & $0.12-0.33$ \\
\hline
\end{tabular}

${ }^{\mathrm{a}} \tau_{\text {eff }}$ as defined in the text.

aries on molecular reorientation. If the effects of motional anisotropy were negligible, then $T_{1}$ would equal $T_{2}$. Since $T_{2} \approx T_{1} / 10$, anisotropic effects dominate the linewidth, although as shown elsewhere [2], the absolute anisotropy, which is measured by $<3 \cos ^{2} \theta^{\prime}-1>$ is still very small.

A second observation that demonstrates that $\tau_{\mathrm{R}}$ is to the left of the $T_{1} \mathrm{~min}$ imum is the temperature dependence of measured $T_{1}$ data. Since $\tau_{\mathrm{R}}$ increases monotonically with temperature, $T_{1}$ exhibits opposite temperature dependence on the two sides of the minimum. Experimentally, the ${ }^{13} \mathrm{C} T_{1}$ data uniformly lengthen with increasing temperature indicating that both epinephrine and the

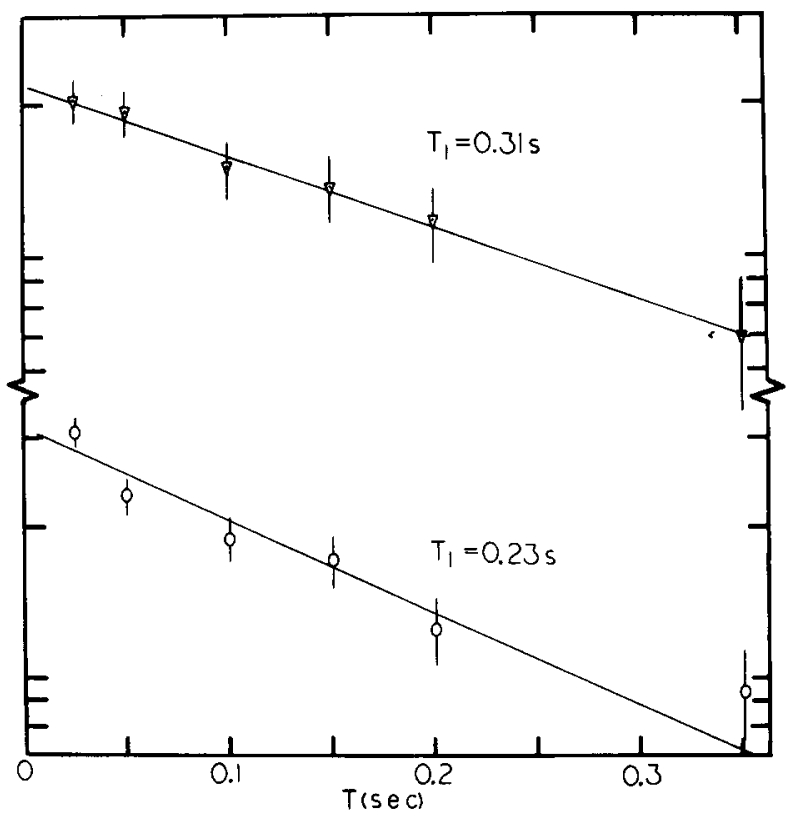

Fig. 2. Semilogarithmic plots of partially relaxed amplitudes: $¥, 13$ C-2 of epinephrine and 0 , the backbone $\alpha^{-13} \mathrm{CH}$ peak of chromogranin. 


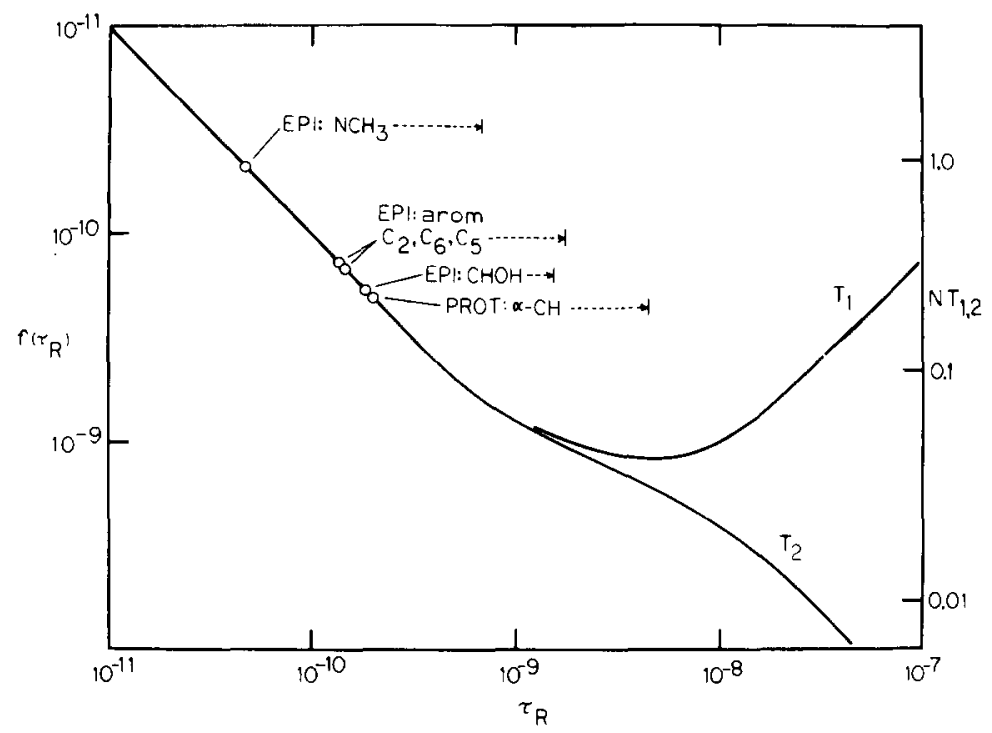

Fig. 3. Reorientational correlation times computed from ${ }^{13} \mathrm{C} T_{1}$ data at $25^{\circ} \mathrm{C}$. Solid lines are theoretical plots of Eqn. 2 of the text and Eqn. 2 of ref. 2 computed for a field strength of 23.49 kG. Dashed arrows indicate upper limits to the correlation times consistent with linewidths reported in ref. 2.

chromogranin backbone have correlation times to the left of the minimum. The $\mathrm{N}^{13} \mathrm{CH}_{3}$ resonance of epinephrine shows little temperature dependence. This behavior is often seen for methyl groups with relatively free internal rotation.

Among the various chromogranin resonances, the $(\alpha-\mathrm{CH})$ carbons overlap to a large extent and give rise to an intense, well defined peak in the methine region. Since the $\alpha$-carbon is protonated it provides a very convenient motional probe of the peptide backbone. Unfortunately, this peak does overlap with the epinephrine $\mathrm{NCH}_{2}$ group which gives rise to a minor portion of the total observed intensity. The epinephrine contribution to this peak is probably $20-$ $25 \%$, assuming that the epinephrine methylene intensity is similar to the intensities of the nearby epinephrine $\mathrm{CHOH}$ and $\mathrm{NCH}_{3}$ groups. In spite of this overlap, the $\alpha-\mathrm{CH}$ peak certainly provides a reasonable measure of the correlation time of the chromogranin peptide backbone. This value, $\tau_{\mathrm{R}}=0.20 \mathrm{ps}$ at $3^{\circ} \mathrm{C}$, is very short and shows beyond question that chromogranin is a random coil protein in a highly fluid medium.

$T_{1}$ data of carbons on the chromogranin sidechains have also been estimated roughly from the null points of two peaks that correspond to the $\beta$ - and $\gamma$-methylene resonances of glutamic acid. $T_{1}$ data of these resonances are uncertain within a factor of two, but they imply correlation times in the same range $\left(\tau_{\mathbf{R}} \sim 0.25 \mathrm{ps}\right)$ as were found for the peptide $\alpha$-carbons and for epinephrine.

Partially relaxed proton spectra of chromaffin granules that were exhaustively exchanged with ${ }^{2} \mathrm{H}_{2} \mathrm{O}$ have also been obtained. $T_{1}$ values for various chemical sites on epinephrine, ATP and chromogranin were measured at 3 and $25^{\circ} \mathrm{C}$ and are listed in Table II. The partially relaxed ${ }^{13} \mathrm{C}$ spectra provide a much more direct method for computing correlation times than do proton spectra, since proton relaxation is not necessarily dominated by nearest-neighbor interac- 
TABLE II

PROTON $T_{1}$ DATA AND CORRELATION TIMES IN CHROMAFFIN GRANULES

\begin{tabular}{|c|c|c|c|c|c|}
\hline \multirow[t]{2}{*}{ Molecule } & \multirow[t]{2}{*}{ Proton } & \multicolumn{2}{|l|}{$3^{\circ} \mathrm{C}$} & \multicolumn{2}{|l|}{$25^{\circ} \mathrm{C}$} \\
\hline & & $T_{1}(\mathrm{~s})$ & ${ }^{\tau_{R}}$ (ns) & $T_{1}(\mathrm{~s})$ & $\tau_{\mathbf{R}}(\mathrm{ns})$ \\
\hline Epinephrine & $\begin{array}{l}\text { aromatic } \\
\mathrm{NCH}_{2} \\
\mathrm{NCH}_{3}\end{array}$ & $\begin{array}{l}0.22 \pm 0.03 \\
0.085 \pm 0.025 \\
0.22 \pm 0.06\end{array}$ & $\begin{array}{l}0.23^{a} \\
0.14^{a} \\
-\end{array}$ & $\begin{array}{l}0.33 \pm 0.04 \\
0.10 \pm 0.03 \\
0.26 \pm 0.07\end{array}$ & $\begin{array}{l}0.15^{a} \\
0.12^{a} ;(<0.27)^{b}\end{array}$ \\
\hline ATP & $\begin{array}{l}\mathrm{H}_{2} \\
\mathrm{H}_{8}\end{array}$ & $\begin{array}{l}0.35 \pm 0.07 \\
0.22 \pm 0.06\end{array}$ & $\begin{array}{c}\overline{1.9}+1.0 \mathrm{a} \\
-0.9\end{array}$ & $\begin{array}{l}0.29 \pm 0.06 \\
0.16 \pm 0.07\end{array}$ & $\begin{array}{c}- \\
1.0+1.0 \mathrm{a} \\
-0.5\end{array}$ \\
\hline Chromogranin & $\begin{array}{l}\mathrm{CH}_{3} \\
\mathrm{CH}_{2}\end{array}$ & $\begin{array}{l}0.14 \pm 0.04 \\
0.10 \pm 0.03\end{array}$ & $\overline{(<0.37)}^{b}$ & $\begin{array}{l}0.17 \pm 0.04 \\
0.10 \pm 0.03\end{array}$ & $\overline{(<0.37}^{b}$ \\
\hline
\end{tabular}

a Correlation times computed by comparison with $T_{1}$ data in a reference solution, using a procedure described in the text.

${ }^{b}$ Upper limits to correlation times computed by considering only the relaxation contribution arising from dipolar couplings of geminal protons.

tions, and reliable values of ${ }_{i}^{\Sigma_{i j}} r_{i j}^{-6}$ may be difficult to obtain. In the present instance, however, the proton spectra provide the only available probe of molecular motion of ATP. Two protons on the adenine base, $\mathrm{H}_{2}$ and $\mathrm{H}_{8}$, are readily monitored and appear downfield of the aromatic resonances of epinephrine in a spectral region that is essentially free of interfering resonances (Fig. 4). Unfortunately, the ribose protons of ATP are obscured by the intense ${ }^{2} \mathrm{HHO}$ peak and the $\alpha-\mathrm{CH}$ peak of chromogranin, and are unsuitable for study. $\mathrm{H}_{2}$ and

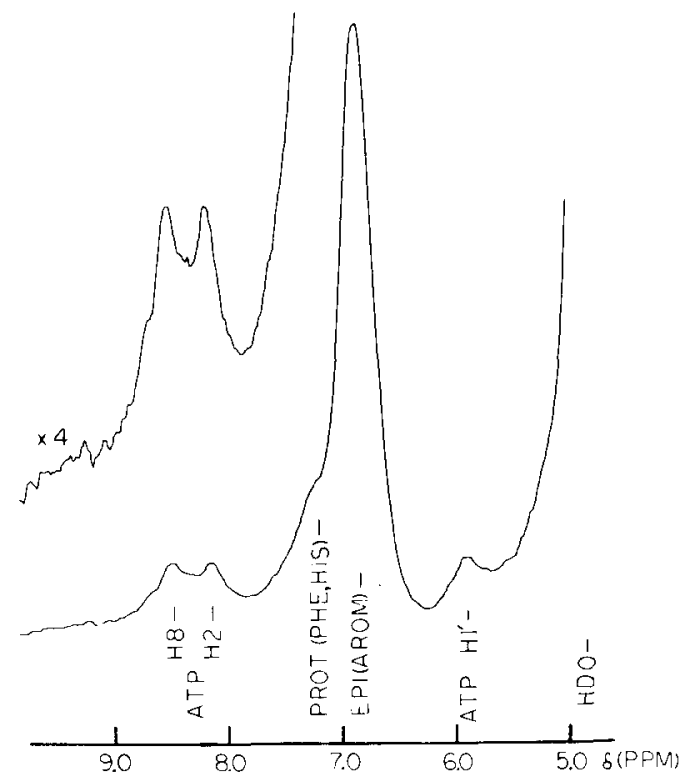

Fig. 4. Proton spectrum of chromaffin granules in the region downfield of the ${ }^{2}$ HHO peak. 
$\mathrm{H}_{8}$, however, are quite informative in that the temperature dependence of their $T_{1}$ data is qualitatively different from that of any of the resonances of epinephrine or chromogranin. Whereas $T_{1}$ data of the latter molecules uniformly lengthen with increasing temperature, $T_{1}$ data of $\mathrm{H}_{2}$ and $\mathrm{H}_{8}$ decrease with increasing temperature. The magnitude of this effect is near the limits of experimental uncertainty but was reproduced on different batches of chromaffin granules. Such behavior is indicative of an ATP correlation time to the right of the $T_{1}$ minimum for protons, or $\tau_{\mathrm{R}} \geqslant 1.0 \mathrm{~ns}$ at $25^{\circ} \mathrm{C}$.

Another way of estimating $\tau_{\mathrm{R}}$ for ATP is to compare the proton $T_{1}$ data in chromaffin granules with the $T_{1}$ data in a reference solution, for which the absolute value of $\tau_{\mathbf{R}}$ can be measured directly by ${ }^{13} \mathrm{C}$ NMR. In this way the ratio of correlation times (or more precisely of the functions $f_{1}\left(\tau_{R}\right)$ ) in the two media is given by the inverse ratio of $T_{1}$ data while the absolute value of $\tau_{R}$ is calibrated by ${ }^{13} \mathrm{C}$ data in the reference solution. In order that the measured correlation time characterize reorientational motion of ATP, however, it is necessary that the monitored proton be relaxed primarily through intramolecular dipolar couplings. This condition should be satisfied quite well by $\mathrm{H}_{8}$ on the adenine base of ATP. In the low energy anti conformation of ATP, this proton sits in a pocket above the furanose ring in close proximity to four ribose protons, $\mathrm{H}_{2}{ }^{\prime}, \mathrm{H}_{3}{ }^{\prime}, \mathrm{H}_{5}{ }^{\prime}$ and $\mathrm{H}_{5}{ }^{\prime \prime}$. Dipolar couplings to these protons almost certainly dominate $T_{1}$ in ${ }^{2} \mathrm{H}_{2} \mathrm{O}$ solutions, where intermolecular relaxation contributions arising from the solvent protons are effectively suppressed.

Using the $\mathrm{H}_{8}$ proton to monitor ATP reorientation, we compute correlation times of $1.0 \mathrm{~ns}$ at $25^{\circ} \mathrm{C}$ and $1.9 \mathrm{~ns}$ at $3^{\circ} \mathrm{C}$ (data from the reference solution are given in Table III). These estimates are about seven times longer than the directly measured correlation times for epinephrine and the chromogranin backbone. The former value is very near the $T_{1}$ minimum (see Fig. 5) and is consistent with both the NOE behavior and the anomalous temperature dependence of ATP resonances cited above. It should be noted, however, that this procedure is indirect in that it "calibrates" the geometric factor, ${ }_{j}^{\Sigma} r_{i j}^{-6}$, by measurements in a reference solution. It could be subject to substantial error if, in the two media, the adenine moiety is in different conformations with respect

\section{TABLE III}

CARBON AND PROTON $T_{1}$ DATA AND MOLECULAR CORRELATION TIMES IN REFERENCE SOLUTIONS CONTAINING EPINEPHRINE (1.0 M IN $2 \mathrm{H}_{2} \mathrm{O}$, DEGASSED) AND ATP $\left(0.5 \mathrm{M} \mathrm{IN} 2 \mathrm{H}_{2} \mathrm{O}\right.$, DEGASSED)

$T_{1}$ data measured at $25 \pm 2^{\circ} \mathrm{C}$.

\begin{tabular}{llllll}
\hline Epinephrine & Proton & $T_{1}(\mathrm{~s})$ & Carbon & $T_{1}(\mathrm{~s})$ & ${ }^{\tau} \mathbf{R}(\mathrm{ns})$ \\
\hline $1.0 \mathrm{M}$ & $\mathrm{CH}_{2} \mathrm{~N}$ & 0.28 & $\mathrm{CH}_{2} \mathrm{~N}$ & 0.55 & 0.042 \\
& $\mathrm{CH}_{3} \mathrm{~N}$ & 0.54 & $\mathrm{CH}_{3} \mathrm{~N}$ & 1.18 & 0.013 \\
& $\mathrm{C}_{2}, \mathrm{C}_{5}, \mathrm{C}_{6}$ & 0.54 & $\mathrm{C}_{2}, \mathrm{C}_{5}, \mathrm{C}_{6}$ & 0.51 & 0.09 \\
& & & $\mathrm{HCOH}$ & 1.17 & 0.04 \\
& & & & & \\
ATP & $\mathrm{H}_{8}$ & 0.27 & $\mathrm{C}_{8}$ & 0.107 & $0.38 \pm 0.04$ \\
$0.5 \mathrm{M}$ & $\mathrm{H}_{2}$ & 0.56 & $\mathrm{C}_{2}$ & 0.099 & $0.41 \pm 0.04$ \\
\hline
\end{tabular}

\footnotetext{
${ }^{a} \tau_{\text {eff }}$ as defined in the text.
} 


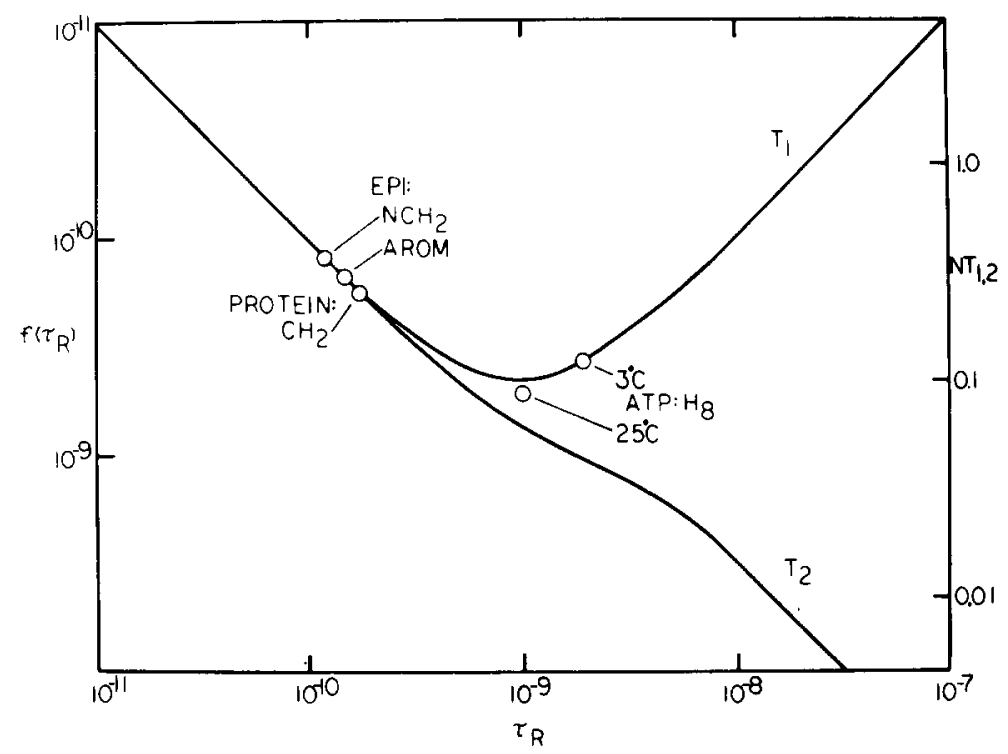

Fig. 5. Reorientational correlation times computed from proton $T_{1}$ data. All data taken at $25^{\circ} \mathrm{C}$ except as otherwise noted. Solid lines are plots of Eqn. 1 of the text and Eqn. 1 of ref. 2, computed for a field strength of $23.49 \mathrm{kG}$. The $\mathrm{NCH}_{3}$ correlation time is $\tau_{\text {eff }}$ as defined in the text.

to the ribose ring. When applied to epinephrine, however, this method gives values of $\tau_{\mathrm{R}}$ that are entirely consistent with directly measured values obtained from the ${ }^{13} \mathrm{C}$ spectra (see Table II and Fig. 5).

The region between 1.40 and $2.40 \mathrm{ppm}$ in the proton spectrum contains four intense peaks due to methylene protons on the amino acid sidechains of chromogranin. Relaxation times of these resonances likewise do not provide a direct probe of sidechain motion, since the factor $\Sigma r_{i j}^{-6}$ in the $T_{1}$ expression contains comparable contributions from dipolar couplings of both geminal and vicinal protons. Only the geminal couplings are accurately known. A rough estimate of the methylene correlation time can be obtained by neglecting the vicinal couplings and using an average $T_{1}$ to describe relaxation in the methylene region (see Table II). This approximation gives $\tau_{\mathrm{R}, \mathrm{av}} \leqslant 0.37 \mathrm{~ns}$. It provides an upper limit on the average correlation time, which it probably overestimates by a factor of about two. It is in reasonable agreement with the range of correlation times, $\tau_{\mathrm{R}}=0.12-0.33 \mathrm{~ns}$, calculated from ${ }^{13} \mathrm{C}$ resonances of the $\beta$ - and $\gamma$-methylene groups of glutamic acid.

\section{Discussion}

Among the various correlation time measurements discussed above, the most accurate are those based on the $T_{1}$ data of several protonated carbons of epinephrine and on the $T_{1}$ data of the $\alpha$-methine carbons of chromogranin. Uncertainty in these correlation times is approx. $\pm 10 \%$ at $25^{\circ} \mathrm{C}$, and $\pm 30 \%$ (due to poorer signal to noise ratio because of the lack of a crystal filter) at $3^{\circ} \mathrm{C}$. All of the epinephrine resonances, with the exception of those on the N-methyl group, which has an internal degree of rotational freedom, indicate a uniform 
reorientational correlation time of $0.15 \mathrm{~ns}$ at $25^{\circ} \mathrm{C}$ and $0.32 \mathrm{~ns}$ at $3^{\circ} \mathrm{C}$ for the catechol moiety and the $\beta$-methine carbon. The proton data in Table II show that $\tau_{\mathrm{R}}$ for the $\mathrm{NCH}_{2}$ group is similar in magnitude. Comparing these correlation times with those in a $1.0 \mathrm{M}$ aqueous solution of epinephrine (Table III), it is evident that the reorientation of epinephrine is slowed by a factor of $2-4$ in the intragranular medium relative to a simple aqueous solution. This difference is surprisingly small. Furthermore, it must account for the combined effects of viscosity changes and molecular immobilization due to specific complex formation in the the chromaffin granule aqueous phase. Thus, the aqueous phase is clearly highly fluid. The viscosity of this phase is at most four times that of water, since in the absence of complex formation, $\tau_{\mathrm{R}}$ varies directly as viscosity over $k T$ [9]. Furthermore, epinephrine almost certainly interacts strongly with the other soluble components. It diffuses freely through the chromaffin vesicle membrane at $0^{\circ} \mathrm{C}$ and yet is maintained indefinitely in the storage granules in an osmotically inactive form in the presence of large concentration gradients $[3,4,12]$. The low osmotic activity of epinephrine in intact vesicles implies some restriction to its rotational motion, and hence the measured $\tau_{R}$ values suggest that the viscosity of the aqueous phase is very similar to that of dilute aqueous solutions.

The fluidity of the medium is also mirrored in the very short correlation times of the chromogranin backbone and sidechains. $\tau_{R}$ for the $\alpha$-methine carbon on the peptide backbone is quite similar to that of the epinephrine catechol moiety (of course this value represents an average for all methine carbons in the peptide chain). Correlation times of methylene groups on the amino acid side chains are known less accurately than that of the $\alpha$-methine carbon, but are in the same general range: $\tau_{R}=0.12-0.33 \mathrm{~ns}$ at $25^{\circ} \mathrm{C}$. These values emphasize the nature of chromogranin as a random coil polyelectrolyte that lacks secondary structure.

Perhaps the most interesting finding is that the correlation time for ATP (as measured by the H-8 proton on the adenine base) appears to be substantially longer than correlation times of either epinephrine or chromogranin. The inference of an ATP correlation time near $1.0 \mathrm{~ns}$ rests on three observations. First, the $T_{1}$ data of both $\mathrm{H}_{2}$ and $\mathrm{H}_{8}$ increase with decreasing temperature, which is possible only if the correlation time is to the right of the $T_{1}$ minimum (i.e. $\tau_{\mathrm{R}} \geqslant 1.0 \mathrm{~ns}$ ). The temperature dependence of the $T_{1}$ data of these protons was measured on two different granule preparations and reproducibly showed qualitatively different behavior than the $T_{1}$ data of other resonances. Secondly, the amplitudes of ATP peaks in the carbon spectra are anomalously low with respect to the amplitudes of epinephrine peaks. The only two obvious reasons for this difference are loss of nuclear Overhauser enhancement (NOE) or differential broadening of ATP resonances relative to epinephrine. Either reason implies a longer correlation time for ATP. Doddrell et al. [11] have calculated the dependence of Overhauser enhancement on $\tau_{\mathrm{R}}$ for ${ }^{13} \mathrm{C}$ resonances at 25.1 MHz. Very little loss of NOE occurs when $\tau_{\mathrm{R}} \tilde{<} 0.4 \mathrm{~ns}$, but the enhancement drops rapidly toward unity in the region $1.0 \mathrm{~ns}<\tau_{\mathrm{R}}<3.0 \mathrm{~ns}$. Thus, from the correlation times in Tables I and II, maximum enhancements are expected for epinephrine and chromogranin but significantly reduced enhancements are expected for ATP. And thirdly, a difference in correlation time for ATP and 
epinephrine is consistent with our comparison of the $T_{1}$ data of $\mathrm{H}_{8}$ in a reference solution and in chromaffin granules.

The conclusion that ATP is significantly less mobile than either epinephrine or chromogranin raises intriguing questions concerning the nature of the intermolecular complexes responsible for the low osmotic activity of epinephrine. Measured correlation times show that chromogranin is a random coil polyelectrolyte and therefore strongly suggest that the complex may be of the type formed between a polyion and its counterions. Electrostatic interactions inside the polyion domain are much larger than those between simple ions and are certainly adequate to lower substantially the osmotic activity of low molecular weight counterions $[13,14]$. It is also natural that epinephrine and ATP would play very different roles in such a complex due to their different charges. Specifically, the multiple negative charges of ATP could lead to site binding of positively charged amino acid sidechains and consequent cross-linking of the protein. Localization of ATP at cross-linked sites would account at least qualitatively for its low rotational mobility relative to epinephrine and the peptide backbone. The possible relevance of such complexes to the hormone storage mechanism will be considered at greater length elsewhere.

\section{Acknowledgements}

This work was supported in part by Institutional Research Grant No. IN-40P to the University of Michigan from the American Cancer Society. Stimulating discussions with Dr. Edward Domino are gratefully acknowledged.

\section{References}

1 Daniels, A., Korda, A., Tanswell, P., Williams, A. and Williams, R.P.J. (1974) Proc. R. Soc. Lond. Ser. B, 187, 353-361

2 Sharp, R.R. and Richards, E.P. (1976) Biochim. Biophys. Acta (to be published)

3 Hillarp, N.-Å. (1958) Acta Physiol Scand, 42, 321-332

4 Hillarp, N.-Â. (1958) Acta Physiol. Scand. 47, 271-279

5 Blaschko, H., Born, C.V.R., D'Iorio, A.D. and Eade, N.R. (1956) J. Physiol. Lond. 133, 548-557

6 Folck, B., Hillarp, N.A. and Högberg, B. (1956) Acta Physiol. Scand. 36, 361-376

7 Helle, K.B. (1966) Mol. Pharmacol. 2, 298-310

8 Slotkin, T.A., Ferris, R.M. and Kirshner, N. (1971) Mol. Pharmacol. 7, 308-316

9 Abragam. A. (1961) The Principles of Nuclear Magnetism, Chapter VIII, Oxford University Press, New York

10 Woessner, D.E. (1962) J. Chem. Phys. 36, 1-4

11 Doddrell, D., Glushko, V. and Allerhand, A. (1972) J. Chem. Phys. 56, 3683-3689

12 Carlsson, A. and Hillarp, N.-A. (1958) Acta Physiol. Scand. 44, 163-169

13 Pals, D.T.F. and Hermans, J.J. (1952) Rec. Trav. Chim. 71, 458-467

14 Rice, S.A. and Nagasawa, M. (1961) Polyelectroly te Solutions, Chapter 8, Academic Press, London 